\title{
Effect of Ions and Ionic Strength on Surface Plasmon Absorption of Single Gold Nanowires
}

Susil Baral ${ }^{1}$, Andrew J. Green ${ }^{1}$, and Hugh H. Richardson ${ }^{1}$ *

${ }^{1}$ Department of Chemistry and Biochemistry, Ohio University, Athens, Ohio 45701

Supporting Information for Publication

\section{SEM Image of the Nanowire}

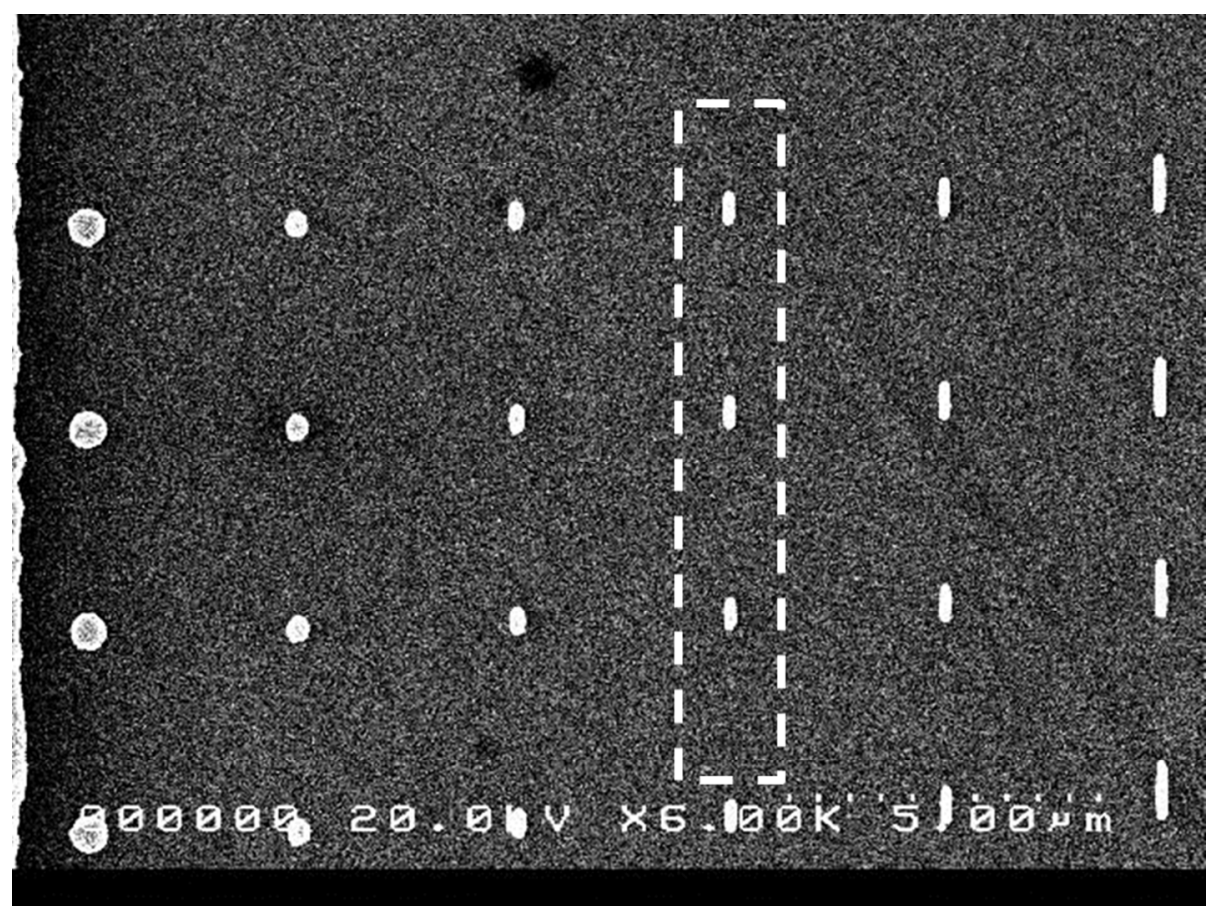

Figure S1. SEM image of the gold nanowires. The nanowires highlighted inside the white dashed lines represent the typical nanowire studied on this work and has typical dimensions of width and length of $200 \pm 8 \mathrm{~nm}$ and $500 \pm 15 \mathrm{~nm}$ respectively. 


\section{Representative Absorption Spectra of a Single Gold Nanorod(s)}
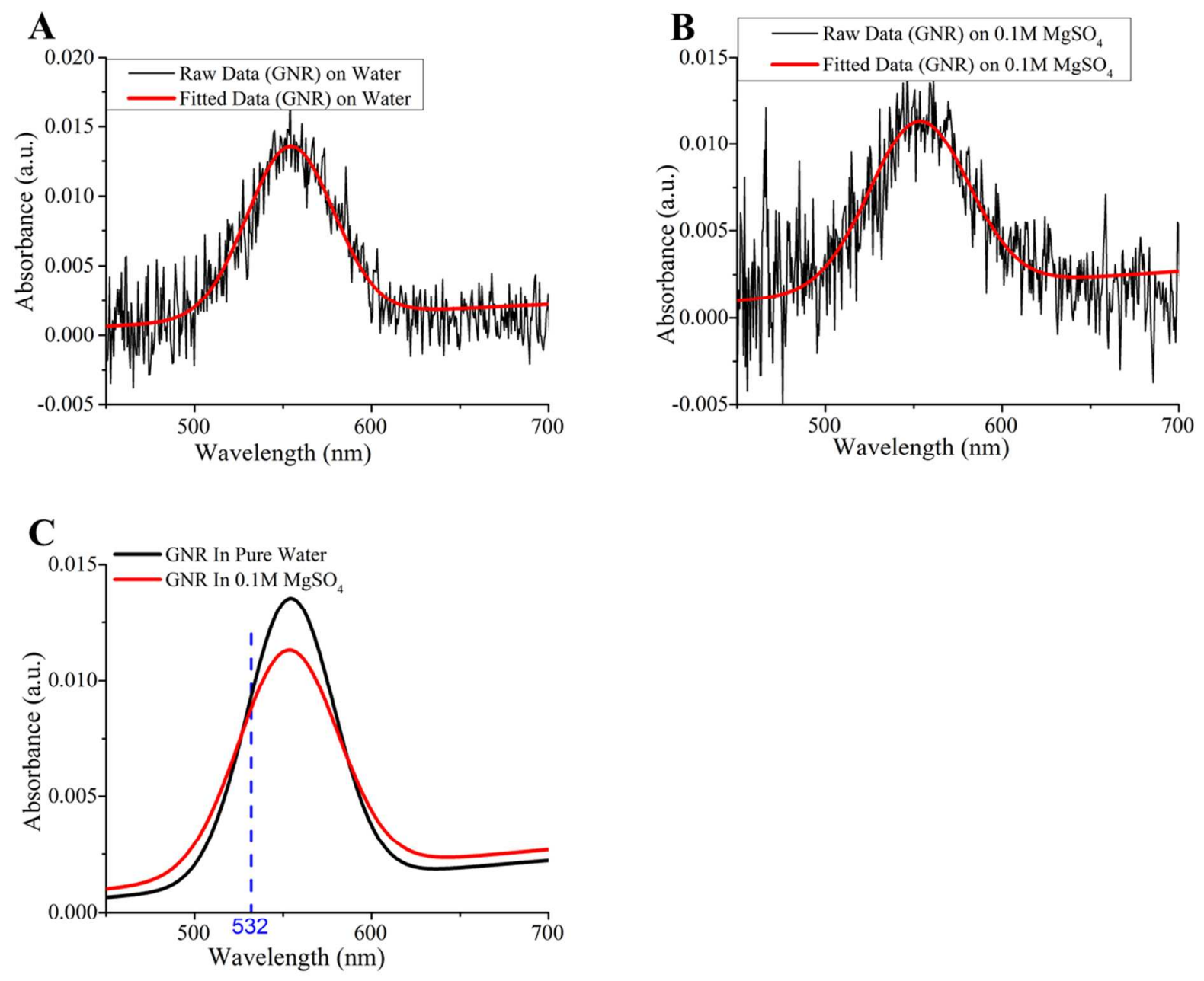

Figure S2. Absorption spectra of a single gold nanorod(s) spin coated onto the glass cover slip substrate under pure water and $0.1 \mathrm{M} \mathrm{MgSO}_{4}$ solution. The fitted spectra (Figure 4C) do not show noticeable shift on the plasmon resonance wavelength but shows clear attenuation on plasmon absorbance of the gold nanorod(s) immersed under ionic solution of $0.1 \mathrm{M} \mathrm{MgSO}_{4}$. 


\section{Representative Absorption Spectra and Thermal Profile of a Single Gold Nanoparticle(s)}
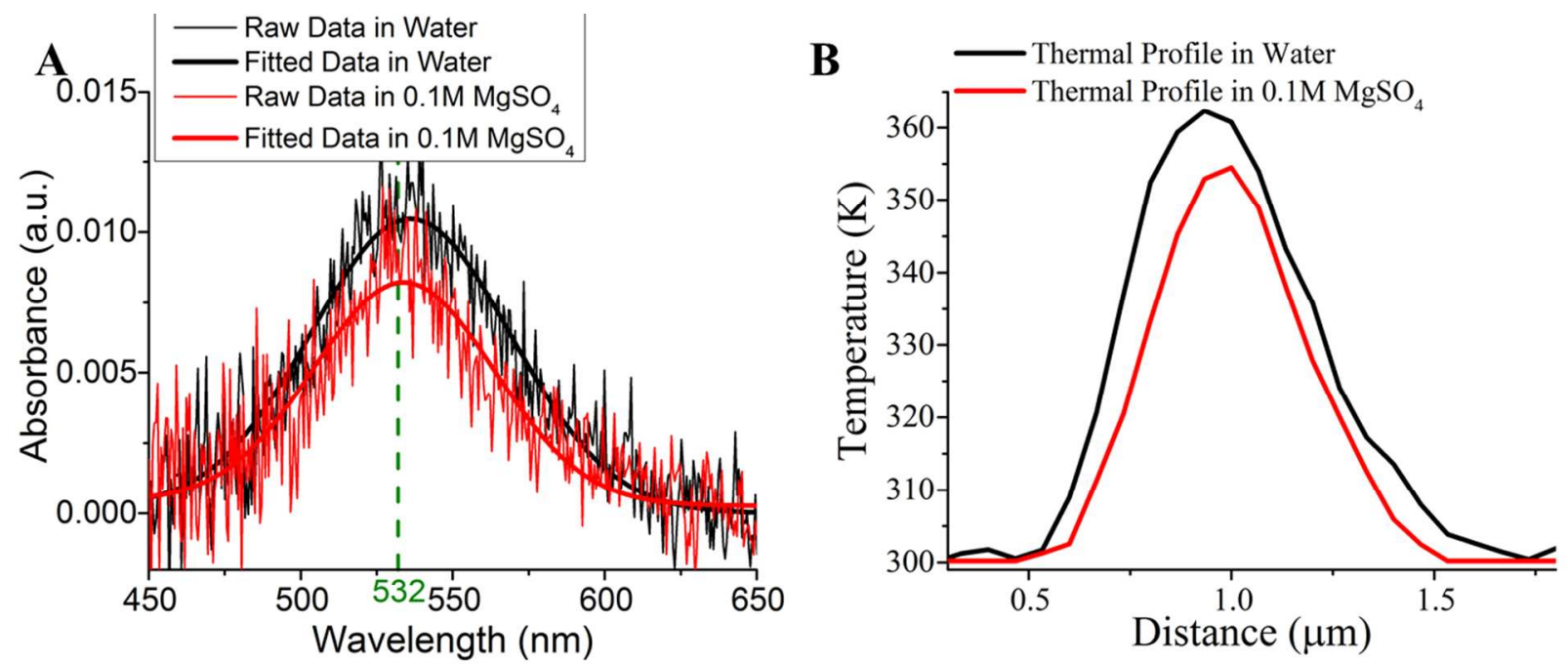

Figure S3. (A) Absorption spectra and (B) the thermal profile under $532 \mathrm{~nm} \mathrm{CW}$ (Intensity $6 \times 10^{9}$ $\mathrm{W} / \mathrm{m}^{2}$ ) excitation of $40 \mathrm{~nm}$ gold nanoparticle(s) spin-coated onto the substrate with thermal sensor film of $\mathrm{AlGaN}: \mathrm{Er}^{3+}$ on sapphire glass. Black spectra represent the absorption spectrum and the corresponding thermal profile for the nanoparticle(s) excitation in pure water. Red spectra represent the absorption spectrum and the corresponding thermal profile for the same nanoparticle(s) in $0.1 \mathrm{M} \mathrm{MgSO}_{4}$ solution. The spectra do not show any noticeable shift on plasmon absorption band but shows attenuation on plasmon absorbance of the nanoparticle(s). At $532 \mathrm{~nm}$, absorption spectrum shows about $16 \%$ attenuation on plasmon absorbance (from 0.00985 to 0.00821$)$ and the corresponding drop in maximum temperature change $\left(\Delta \mathrm{T}_{\max }\right)$ is about 14\% (drop from 363 to 354 ). 
Single Particle(s) Absorption, Dark Field Scattering and Plasmon Emission Images
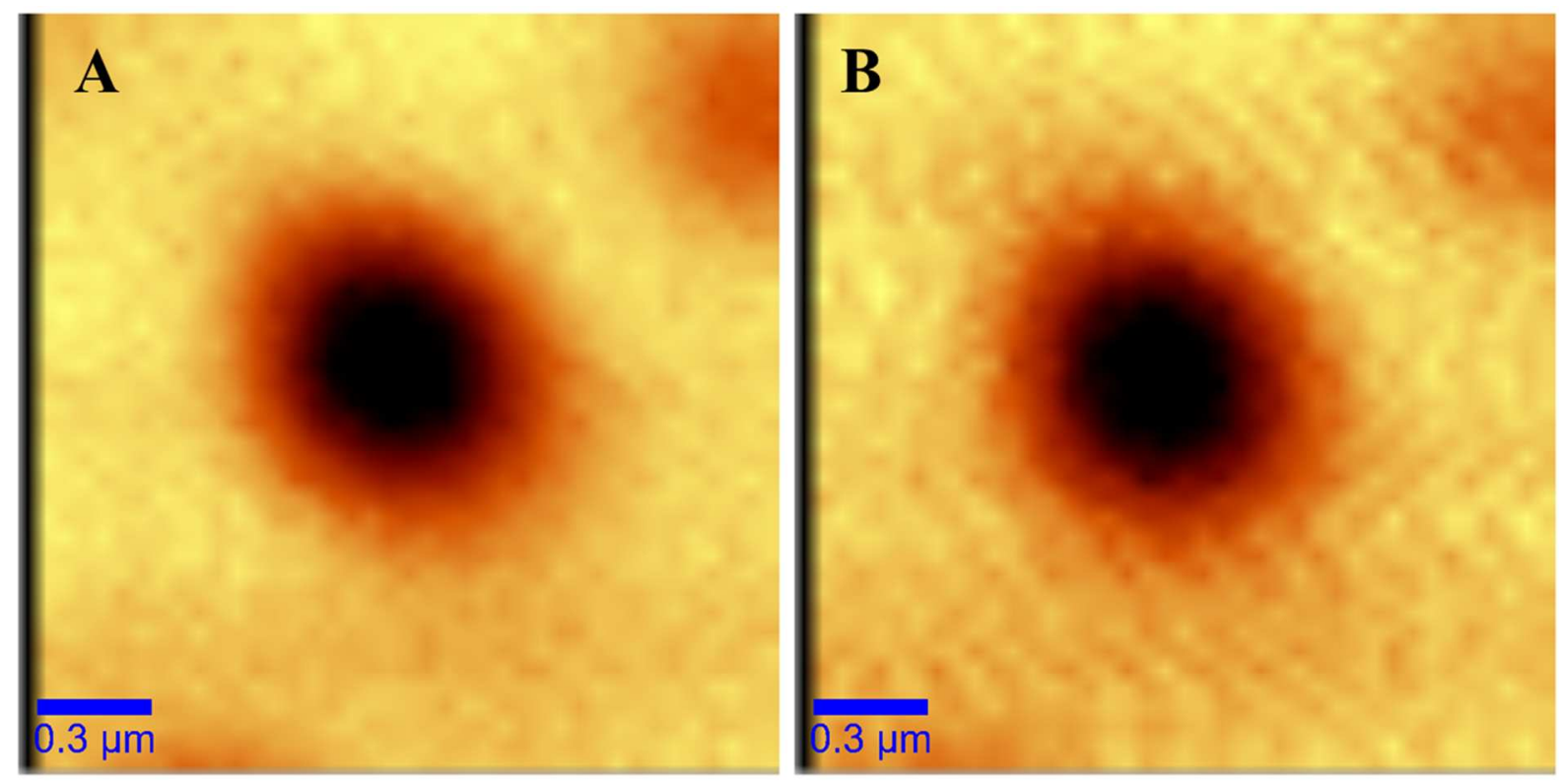

Figure S4. Absorption Images of a single gold nanoparticle(s) spin coated onto the glass cover slip substrate under (A) pure water and (B) $0.1 \mathrm{M} \mathrm{MgSO}_{4}$ solution.

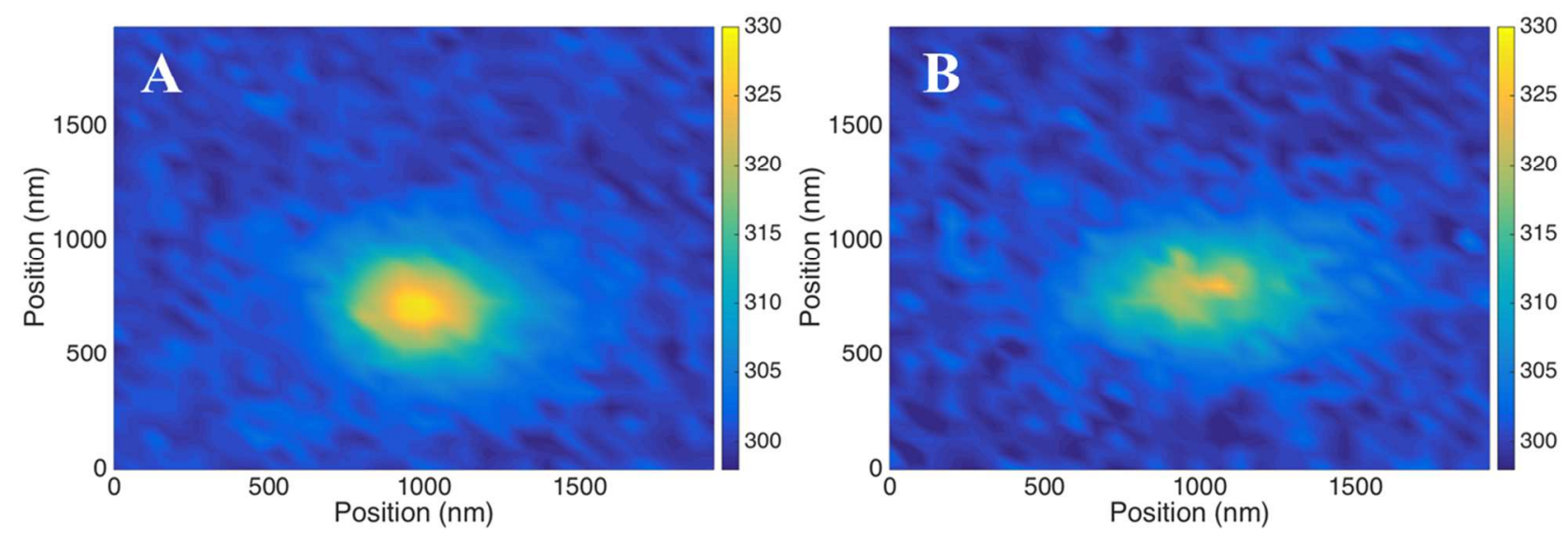

Figure S5. Thermal images of a single optically excited gold nanoparticle(s) spin coated onto the substrate with thermal sensor film of $\mathrm{AlGaN}: \mathrm{Er}^{3+}$ on sapphire glass in (A) pure water and (B) 0.1 $\mathrm{M} \mathrm{MgSO}_{4}$ solution. 

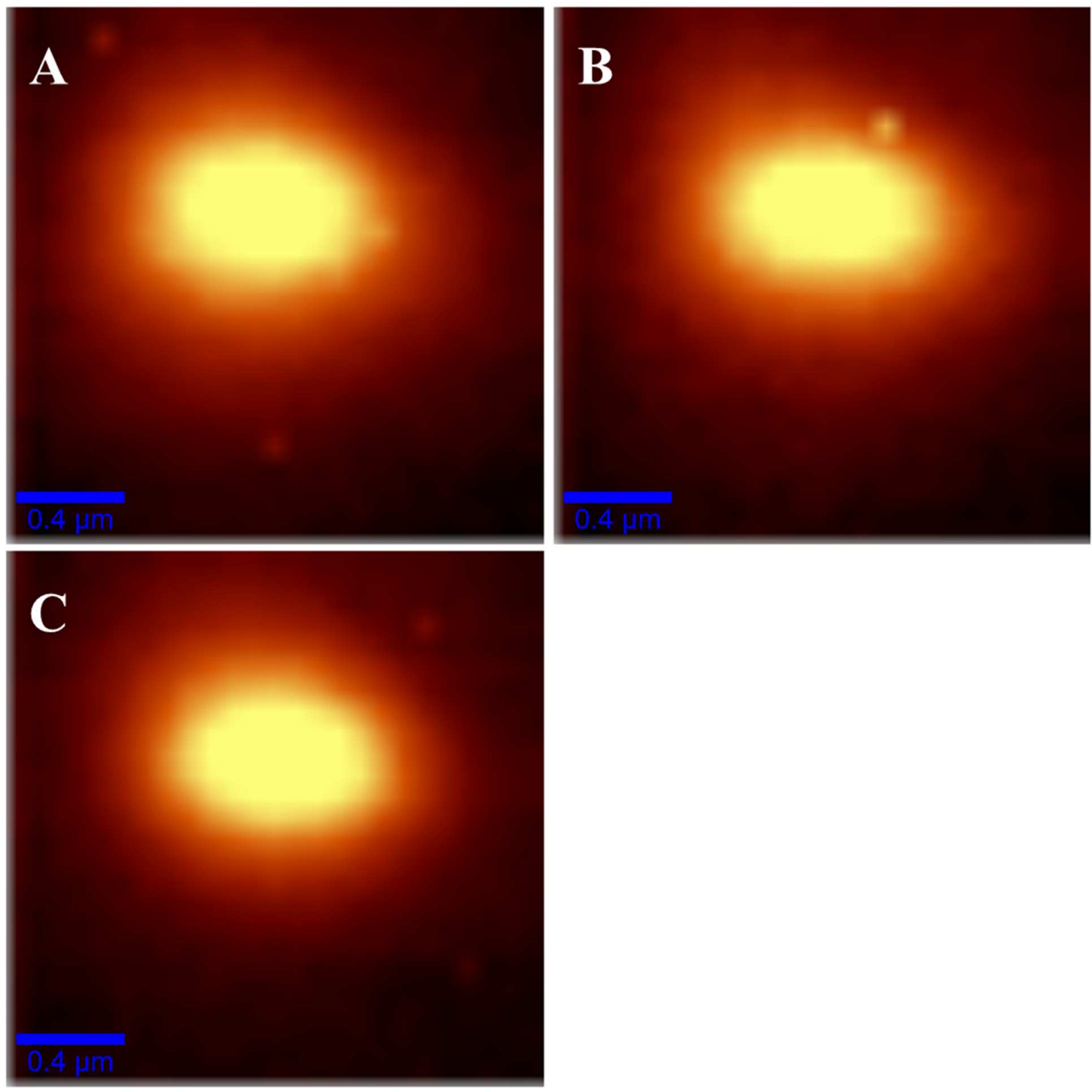

Figure S6. Dark-Field scattering images of a single lithographic gold nanowire under (A) pure water, (B) $0.1 \mathrm{M} \mathrm{NaCl}$ and (C) $0.1 \mathrm{M} \mathrm{MgSO}_{4}$ solution. 


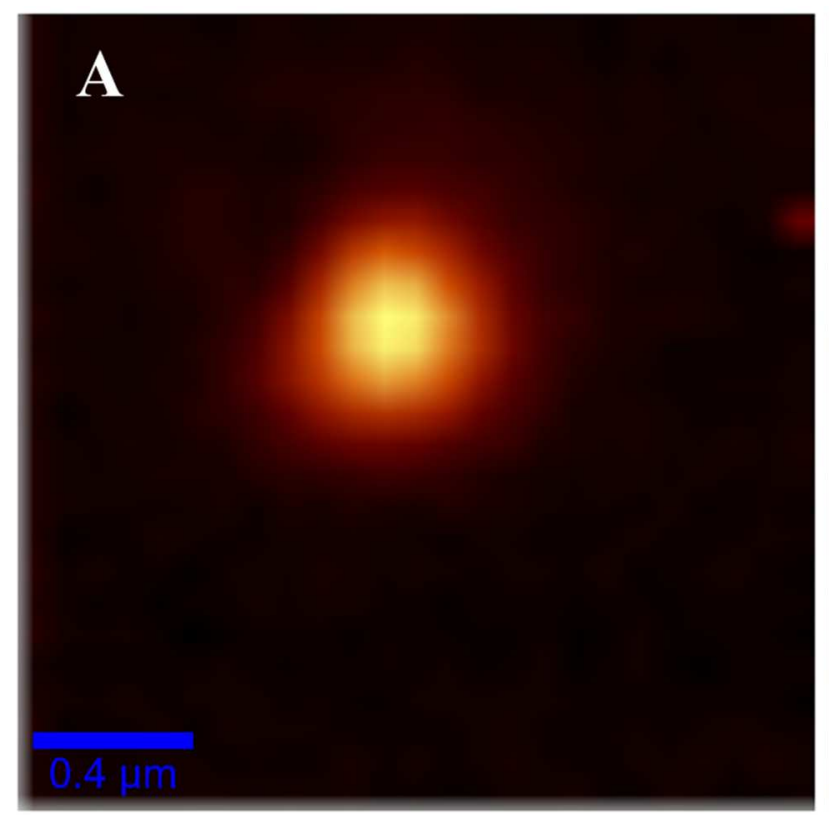

\section{B}

Figure S7. Plasmon emission images of a single gold nanoparticle(s) spin coated onto the glass cover slip substrate under $533 \mathrm{~nm} \mathrm{CW}$ excitation in (A) pure water and (B) $0.1 \mathrm{M} \mathrm{MgSO}_{4}$ solution. 